\title{
Comment faire face à l'éco-anxiété : 11 stratégies d'adaptation en contexte éducatif
}

Marie-Élaine Desmarais, Rhéa Rocque et Laura Sims

\section{OpenEdition}

1 Journals

Édition électronique

URL : https://journals.openedition.org/ere/8267

DOI : 10.4000 /ere.8267

ISSN : 2561-2271

Éditeur

Centr'ERE

Référence électronique

Marie-Élaine Desmarais, Rhéa Rocque et Laura Sims, « Comment faire face à l'éco-anxiété : 11 stratégies d'adaptation en contexte éducatif », Éducation relative à l'environnement [En ligne], Volume 17-1 | 2022, mis en ligne le 27 janvier 2022, consulté le 04 février 2022. URL : http:// journals.openedition.org/ere/8267 ; DOI : https://doi.org/10.4000/ere.8267

Ce document a été généré automatiquement le 4 février 2022

La revue Éducation relative à l'environnement est mise à disposition selon les termes de la Licence Creative Commons Attribution - Pas d'Utilisation Commerciale 4.0 International. 


\title{
Comment faire face à l'éco-anxiété : 11 stratégies d'adaptation en contexte éducatif
}

\author{
Marie-Élaine Desmarais, Rhéa Rocque et Laura Sims
}

\section{NOTE DE L'AUTEUR}

Un webinaire sur ces stratégies a été réalisé par les mêmes auteures : https:// www.loom.com/share/e2fa6daec23f45058303bof68974f994

1 Au cours des dernières années, la crise climatique, ses défis et ses conséquences interreliés (en particulier, les évènements météorologiques extrêmes, la perte de biodiversité, les inégalités persistantes) et le rôle de l'éducation relative à l'environnement à cet effet ont fait de plus en plus l'objet de discussions sociétales (Pihkala, 2020). Les défis relatifs aux changements climatiques sont de plus en plus importants (IPCC, 2018) et préoccupent un nombre grandissant d'individus (Desveaux, 2020). Par ailleurs, la crise climatique est considérée comme la plus importante menace à la santé publique mondiale (Watts et coll., 2015). C'est ainsi que les enjeux climatiques ont des impacts non seulement sur la planète et l'économie, mais aussi sur la santé physique et mentale de la population mondiale (Clayton, 2020). Alors que certains ressentent une urgence d'agir et reconnaissent comme une responsabilité sociale le fait de prendre des actions concrètes dans l'espoir de gérer la crise (Desveaux, 2020), d'autres doutent encore que la crise climatique est bien réelle. Devant le peu d'actions mondiales concertées pour lutter contre les changements climatiques, il n'est pas surprenant de voir émerger des nouvelles formes d'émotions affectant directement la vie des individus et qui sont regroupées sous le terme éco-anxiété (Desveaux, 2020). C'est à cet aspect que cet article s'intéresse. D'abord, une brève définition de l'écoanxiété sera présentée. Ensuite, onze stratégies d'adaptation en lien à l'éco-anxiété relative aux changements climatiques seront proposées de manière à outiller les 
personnes agissant dans le milieu scolaire à mieux intervenir auprès de leurs élèves. Finalement, un exemple d'enseignement à cet effet sera présenté.

\section{Mieux comprendre l'éco-anxiété}

2 Le terme éco-anxiété a fait son apparition dans les écrits scientifiques très récemment pour désigner les émotions difficiles vécues par les individus conscients et craintifs d'un effondrement climatique, soit la peur que les changements climatiques dégradent la planète de manière irrémédiable (Pihkala, 2020). Bien que plusieurs termes et définitions soient proposés, l'éco-anxiété correspond généralement à un sentiment de détresse face au dérèglement des écosystèmes. Elle « se situe entre la peur et l'angoisse, s'exprime par un sentiment de perte de confiance envers l'environnement écologique et peut trouver écho dans la crainte de se retrouver désemparé, saisi par un état de détresse agissant de manière lancinante" (Desveaux, 2020, p. 108). L'éco-anxiété englobe donc une variété d'émotions difficiles comme des inquiétudes, de la peur, du désespoir et de l'impuissance (Clayton, 2020). Ces émotions sont généralement causées par le fait que les actions individuelles actuelles, bien que pertinentes et nécessaires, n'ont pas les effets espérés par la personne qui les mènent. De plus, elles apparaissent généralement lorsqu'une personne devient plus informée sur les impacts des changements climatiques à l'échelle planétaire et qu'elle réalise qu'il reste peu de temps pour agir. Cette forme d'anxiété est complexe puisque la crise climatique et tous ses défis inter-reliés représentent une menace dans un futur plus ou moins rapproché pour laquelle les stratégies d'adaptation au stress habituelles ont peu d'impacts (Davenport, 2017 ; Pihkala, 2020 ; Sims, Rocque et Desmarais, 2020). Effectivement, les stratégies d'adaptation au stress visent généralement à gérer une situation immédiate ou à très court terme. Dans le cas des changements climatiques, les actions individuelles conduites sur-le-champ ont peu d'impacts sur le long terme, et donc soulagent peu la personne éco-anxieuse (Sims et coll., 2020). Ainsi, il est donc primordial de s'intéresser à des stratégies d'adaptation qui visent explicitement à intervenir sur l'éco-anxiété.

\section{Onze stratégies d'adaptation à mettre en œuvre}

3 Considérant que la crise climatique est la plus importante que nous vivons actuellement (Watts et coll., 2015), il importe que les acteurs du milieu éducatif soient mieux outillés pour l'aborder. En effet, il est important de se rappeler que le simple fait d'être informé sur les enjeux climatiques peut mener à l'éco-anxiété. Il est donc primordial d'être outillé pour gérer cette éco-anxiété (Sims et coll., 2020). C'est pourquoi onze stratégies d'adaptation spécifiques à l'éco-anxiété seront ici présentées selon trois axes prioritaires : les émotions, le problème et la signification.

\section{Stratégies d'adaptation axées sur les émotions}

Les stratégies d'adaptation axées sur les émotions s'intéressent à la régulation des émotions difficiles qui émanent de la situation d'impuissance face aux changements climatiques (Desveaux, 2020). Ces stratégies permettent à la personne éco-anxieuse de nommer et de comprendre ses émotions, et de reconnaître leur légitimité. En ce sens, il 
faut, premièrement, offrir des occasions aux personnes d'explorer leurs émotions tant de manière individuelle qu'en groupe. Il importe ici de reconnaître que ce n'est pas un exercice facile et il faut souligner et féliciter le courage de ceux et celles qui acceptent d'examiner et de partager ces émotions difficiles. Deuxièmement, il faut, rappeler aux personnes que leurs émotions sont normales et que ce qu'elles vivent est justifié. Cette validation de l'expérience est nécessaire pour leur permettre de mieux gérer leur écoanxiété et de s'adapter. Cela peut les aider également à donner un sens à leur expérience, ce qui est un vecteur d'engagement important (Ojala, 2012). Troisièmement, il importe d'offrir aux personnes diverses opportunités de discussions afin d'échanger sur les émotions. Durant ces moments, tous et toutes doivent pouvoir échanger librement sur les émotions ressenties et être invité.e.s à faire un bilan (Sims et coll., 2020). Quatrièmement, il est nécessaire que les personnes qui animent les échanges offrent des opportunités aux personnes participantes de prendre soin d'ellesmêmes et de respecter les occasions où elles sentent le besoin d'aller à l'extérieur (Jickling et Blenkinsop, 2020). La pleine conscience est souvent utilisée en guise d'écothérapie puisqu'elle aide à se centrer dans l'ici et maintenant et à prendre conscience des émotions vécues. À cet effet, les recherches dans le domaine de la psychologie montrent que ces stratégies d'adaptation liées aux émotions contribuent à une bonne santé mentale, augmentent la concentration et stimulent la créativité (Davenport, 2017 ; Ojala, 2012).

\section{Stratégies d'adaptation axées sur les problèmes}

5 Les stratégies d'adaptation axées sur les problèmes encouragent les élèves à agir. La prise d'action offre une perception de contrôle sur un problème et contribue à la réduction de l'anxiété (Davenport, 2017 ; Ojala, 2012). Puisque la crise climatique est un phénomène complexe et d'ampleur, les actions individuelles ont un effet limité. Il est alors nécessaire de reconnaître cette limite et de l'accepter. Ainsi, la première stratégie centrée sur les problèmes associés à la crise climatique consiste à décomposer ceux-ci et à identifier des pistes d'actions concrètes et réalistes. Ces actions - aussi simples que possible -, visent à augmenter le sentiment d'auto-efficacité des personnes pour réduire leur anxiété. La deuxième stratégie est d'informer les personnes. Il s'agit ici de leur offrir de l'information de qualité, à jour et vulgarisée, de manière à ce qu'elles puissent bien la saisir. Lorsqu'une menace n'est pas suffisamment comprise, elle peut être source d'anxiété, alors que lorsqu'on en saisit les principaux aspects, il est plus simple de s'engager, puisque l'on comprend où et comment il est possible d'agir. Enfin, la troisième stratégie axée sur les problèmes correspond à offrir des occasions aux personnes de poser des actions concrètes locales de manière individuelle ou collective. La prise d'action est un moyen efficace pour lutter contre l'éco-anxiété puisqu'elle permet de s'adapter à la situation en constatant les répercussions immédiates, même à petite échelle.

\section{Stratégies d'adaptation axées sur la signification}

6 Les stratégies d'adaptation axées sur la signification visent la recherche de sens en lien avec la crise climatique et ses défis inter-reliés. Trop souvent la crise climatique est considérée comme décourageante, triste et dramatique. Cette conception négative, bien que réaliste, contribue à une santé mentale précaire et à un désengagement dans la 
lutte pour le climat (Ojala, 2012). C'est pourquoi il est premièrement nécessaire de recadrer la crise et d'y trouver un sens positif. On peut y arriver en étant créatif, novateur et en transformant certaines habitudes de vie. Deuxièmement, il faut offrir aux élèves des occasions pour réimaginer ou réinventer leur futur désiré. Il faut leur donner des opportunités de rêver à ce que peut représenter un avenir durable et positif. Il s'agit en fait d'une forme de visualisation d'un idéal à atteindre, une technique efficace pour réduire l'anxiété (Davenport, 2017). Troisièmement, il est important de fournir des exemples positifs aux élèves de manière à les inspirer. Ces exemples peuvent porter sur des actions locales durables ou sur les actions d'activistes environnementaux inspirants, comme Greta Thunberg. Ils peuvent contribuer à garder espoir et favoriser l'engagement dans la lutte environnementale (Ojala, 2012).

Le tableau suivant offre une synthèse de chacune des stratégies mentionnées ici.

Tableau 1. Stratégies d'adaptation pour outiller les acteurs du milieu scolaire à mieux intervenir auprès de leurs élèves.

\begin{tabular}{|c|c|c|}
\hline \multicolumn{3}{|c|}{ Stratégies d'adaptation axées sur } \\
\hline les émotions & le problème & la signification \\
\hline $\begin{array}{l}\text { 1. Légitimer et explorer } \\
\text { l'expérience. } \\
\text { 2. Valider l'expérience vécue. } \\
\text { 3. Discuter. } \\
\text { 4. Développer du soutien } \\
\text { social. } \\
\text { 5. Prendre soin de soi et passer } \\
\text { du temps dehors. }\end{array}$ & $\begin{array}{l}\text { 6. Décomposer le problème } \\
\text { et identifier des actions } \\
\text { concrètes. } \\
\text { 7. Fournir de l'information. } \\
\text { 8. Encourager les actions } \\
\text { locales. }\end{array}$ & $\begin{array}{l}\text { 9. Trouver un sens } \\
\text { positif. } \\
\text { 10. Réimaginer le futur } \\
\text { 11. Donner des exemples } \\
\text { positifs. }\end{array}$ \\
\hline
\end{tabular}

8 Les stratégies d'adaptations axées sur les émotions, le problème et la signification sont relativement simples à mettre en œuvre dans un contexte éducatif et visent à préserver une bonne santé mentale et améliorer le bien-être en contexte de crise climatique. Afin de bien comprendre chacun de ces éléments, un exemple est présenté à la section suivante.

\section{Un exemple de cette mise en œuvre}

9 Le tableau 2 propose un exemple où les stratégies d'adaptation pourraient être mises en œuvre en contexte éducatif, et ce, dans plusieurs disciplines. Ces stratégies peuvent être utilisées pour aborder le sujet général de la crise climatique ainsi que des sujets spécifiques liés à cette crise (par exemple, la surconsommation). Ces stratégies doivent être adaptées à l'âge des élèves. 
Tableau 2. Exemple de mise en œuvre des stratégies d'adaptation pour l'éco-anxiété

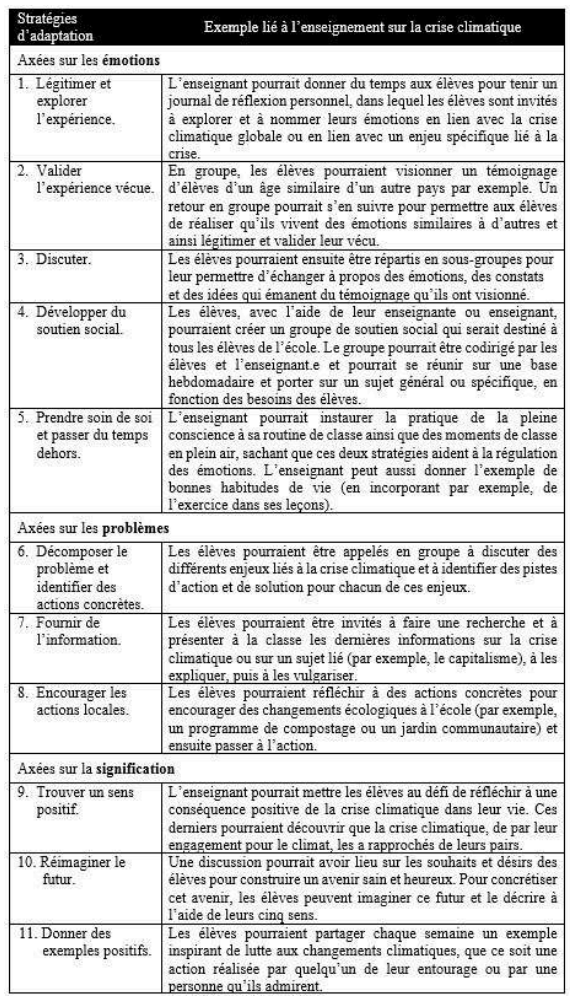

\section{Conclusion}

10 Il semble donc que pour lutter adéquatement contre les changements climatiques, les acteurs des milieux scolaires aient un rôle de premier plan à jouer. Pour exercer efficacement ce rôle, ces derniers doivent poursuivre leurs efforts de sensibilisation, d'information et d'éducation sur les enjeux climatiques et l'urgence d'agir. Ils doivent mettre en œuvre des stratégies pédagogiques qui valorisent le développement de la pensée critique et de l'action citoyenne. Toutefois, ils doivent être conscients que l'éducation aux changements climatiques peut causer de l'anxiété. Bien que l'écoanxiété soit un problème sérieux pouvant causer des effets indésirables sur la santé mentale, elle est aussi un vecteur de changement dans la lutte pour le changement climatique (Pihkala, 2020). Cela dit, trop d'anxiété pourrait paralyser les individus qui en sont atteints, et trop peu d'éco-anxiété pourrait causer un désengagement de la lutte aux changements climatiques (Pihkala, 2020). Il apparaît alors qu'il faut apprendre à vivre avec un certain degré d'éco-anxiété pour s'y engager adéquatement (Davenport, 2017). C'est pour cette raison que les différents acteurs du milieu scolaire doivent être mieux outillés pour intervenir auprès de leurs élèves éco-anxieux. Les onze stratégies d'adaptation proposées dans cet article peuvent être un élément de réponse, tout en reconnaissant que la lutte aux changements climatiques est loin d'être simple et qu'elle interpelle de façon urgente des transformations majeures au sein de nos sociétés. 


\section{BIBLIOGRAPHIE}

Clayton, S. (2020). Climate anxiety : psychological responses to climate change. Journal of Anxiety

Disorders, 74, 102263.

Davenport, L. (2017). Emotional resiliency in the era of climate change : A clinician's guide. Londres : Jessica Kingsley Publishers.

Desveaux, J. (2020). La crainte de l'effondrement climatique : Angoisses écologiques et incidences sur la psyché individuelle. Le Coq-héron, 242(3), 108-115.

The International Panel on Climate Change (IPCC). (2018). Global warming of $1.5^{\circ} \mathrm{C}$. An IPCC Special Report on the impacts of global warming of $1.5^{\circ} \mathrm{C}$ above pre-industrial levels and related global greenhouse gas emission pathways, in the context of strengthening the global response to the threat of climate change, sustainable development, and efforts to eradicate poverty. Geneva, Switzerland : World Meteorological Organization. Repéré à : https://www.ipcc.ch/sr15/

Jickling, B. et Blenkinsop, S. (2020). Wilding teacher education : responding to the cries of nature. Canadian Journal of Environmental Education, 23(1), 121-138.

Ojala, M. (2012). Regulating worry, promoting hope : how do children, adolescents, and young adults cope with climate change. International Journal of Environmental \& Science Education, 7(4), 537-561.

Pihkala, P. (2020). Eco-anxiety and environmental education, Sustainability, 12, 10149.

Sims, L., Rocque, R. et Desmarais, M-É. (2020). Enabling students to face the environmental crisis and climate change with resilience : inclusive environmental and sustainability education approaches and strategies for coping with eco-anxiety. International Journal of Higher Education and Sustainability. 3(1), 1-17.

Watts, N., Adger, W.N., Agnolucci, P., Blackstock, J., Byass, P., Cai, W. et coll. (2015). Health and climate change : policy responses to protect public health. Lancet, 386(10 006), 1861-914.

\section{RÉSUMÉS}

Les défis soulevés par les changements climatiques sont de plus en plus importants et préoccupent un nombre grandissant d'individus. Certains ressentent une urgence d'agir, comme une responsabilité sociale : il faut mener dès maintenant des actions concrètes dans l'espoir de gérer la crise. En raison du fait que les actions individuelles, bien que pertinentes et nécessaires, n'ont pas les effets espérés par la personne qui les mènent, plusieurs vivent de l'éco-anxiété. Afin de canaliser cette écoanxiété, cet article présente onze stratégies d'adaptation, en fonction de trois axes prioritaires : les émotions, le problème et la signification.

The problems caused by climate change are more and more frequent and affect a large number of individuals. Some feel an urgency to take concrete actions in the hope of contributing to manage the crisis : it is a matter of social responsibility Due to the fact that individual actions, while relevant and necessary, do not have the effects hoped for by the person carrying them out, many experience eco-anxiety in response to this crisis. That is why several adaptation strategies specific to eco-anxiety will be presented here according to three different types, those focusing on emotions, the problem and meaning. Each of these strategies will be presented, and an example will be provided. 


\section{INDEX}

Mots-clés : bien-être, éco-anxiété, éducation pour un avenir viable, éducation relative à

l'environnement

Keywords : well-being, eco-anxiety, education for a sustainable future, environmental education

\section{AUTEURS}

\section{MARIE-ÉLAINE DESMARAIS}

Marie-Élaine Desmarais est professeure agrégée à la Faculté d'éducation de l'Université de SaintBoniface à Winnipeg au Manitoba. Elle enseigne des cours liés essentiellement à l'éducation inclusive et aux méthodologies de recherche. Ses recherches portent sur la pédagogie universelle, le bien-être à l'école, la dénormalisation et l'étude du handicap.

\section{RHÉA ROCQUE}

Rhéa Rocque est stagiaire postdoctorale à l'Université de Winnipeg au Manitoba où elle collabore avec le Prairie Climate Centre sur des projets de recherche portant sur les effets du changement climatique sur la santé. Ses recherches actuelles se situent dans le domaine de la psychologie du changement climatique, avec un accent particulier sur la communication climatique et la relation entre la santé et le changement climatique.

\section{LAURA SIMS}

Laura Sims est professeure agrégée à la Faculté d'éducation de l'Université de Saint-Boniface. Elle enseigne, entre autres, les cours liés à la diversité culturelle en éducation, aux perspectives autochtones et à l'éducation relative à l'environnement. Ses recherches portent sur l'éducation relative à l'environnement en contexte d'apprentissage formel et non formel. 